\title{
Graffiti et prostitution
}

Jeanne Demers, Line McMurray et Josée Lambert, photographe

\section{Graffiti and Prostitution}

Urban graffiti constitute a marginal discourse wherein the fears, anxieties, rages and philosophies of city dwellers are clandestinely shared. Graffiti by Montreal prostitutes on a wall in the heart of their district symbolically stake out a territory and are privately coded. In Josée Lambert's photos, we see that these graffiti function in a closed circle: lipstick or make-up pencils (the tools of the trade) have been used to inscribe messages which incite no polemic. The information they offerdescriptions of a john, license plate, danger he presents, etc. - is written to help a sister (to survive?) in the spirit of the collaborative conversations responding to a problem, that are commonly found in women's washrooms. Here, however, reality outstrips fiction, forcing the viewer to see the tragedy in these graffiti, tragedy all the greater for being banalized.

Every graffito can [...] be seen and/or read as a miniature autobiography of a member of a society in the sense that the graffitist reveals a part of himself and his society in all that he writes. ${ }^{1}$

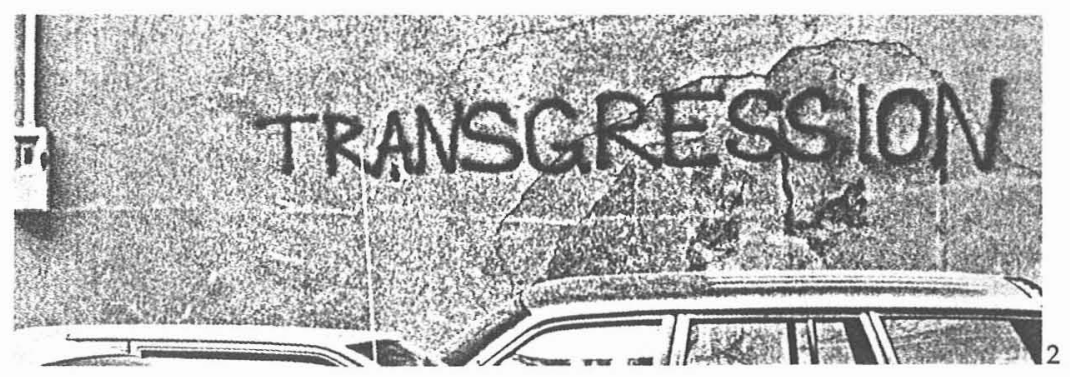


La mode est au graffito urbain. Quelle ville en effet pourrait prétendre échapper au discours marginal que ce dernier entretient jour après jour - ou plutôt nuit après nuit, le phénomène étant forcément clandestin-sur les supports les plus inattendus: blocs de ciment, murs de brique ou de béton, clôtures, vitres, trottoirs, portes, boîtes aux lettres etc. Et toujours il s'agit de crier à la face du monde ses peurs, ses angoisses, ses frustrations, sa colère, de partager avec les éventuels lecteurs l'ironie d'une situation, une philosophie de la vie ou encore la simple joie d'un vers efficace.

Les exemples abondent à Montréal: de

"femmes sans peui"

à

"ma vitre est un jardin de givre Quesque $\mathcal{L}_{e}$ Spasme devirre en passant par

"Trapped", "vive lanarchie cibole", "who's rule

"CET ÉTÉ ON BRONZE MIEUX A TCHEM mObYl"

et

"recréer le réel sous un même Soleil...".

Il arrive que certains graffiti servent symboliquement à marquer un territoire. C'est le cas des occurences imputables aux 'gangs', de quelque obédiance qu'ils soient. ${ }^{4} \mathrm{D}^{\prime}$ autres vont plus loin et choisissent l'exclusivité d'un auditoire privilégié, généralement constitué de pairs. Les graffiti sont alors plus difficiles à repérer à cause, d'une part, de leur discrétion relative-ils n' ont ni à forcer le regard du passant, ni à produire sur lui un quelconque effet de séduction d'autre part, d'un contenu qui ne fait sens que pour des initiès.

Aussi a-t-il fallu un heureux hasard pour que nous découvrions le phénomène sur des panneaux situés angle Dorchester Est et Hôtelde-Ville, soit en plein coeur d'un quartier de prostitution (photo 1). 

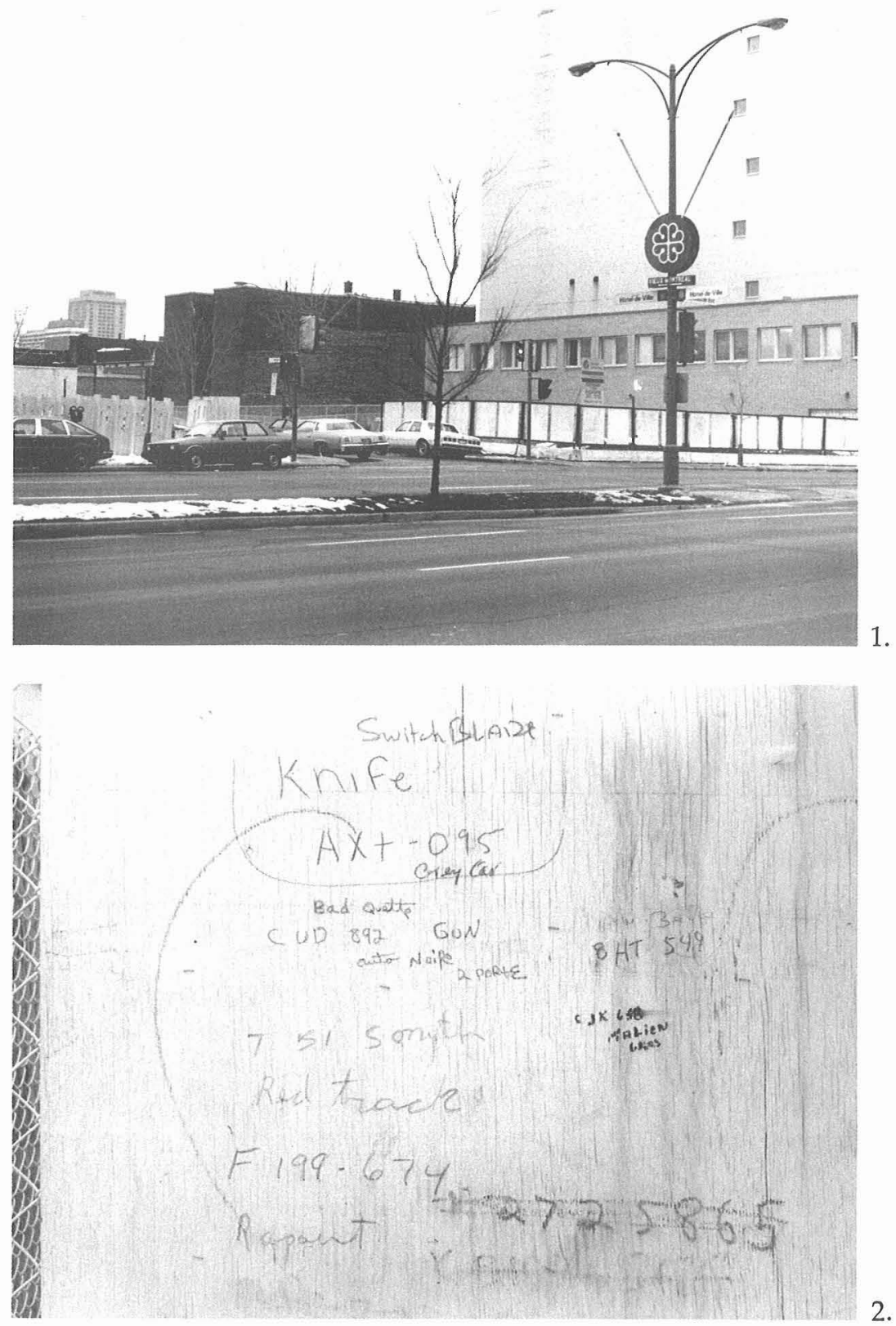


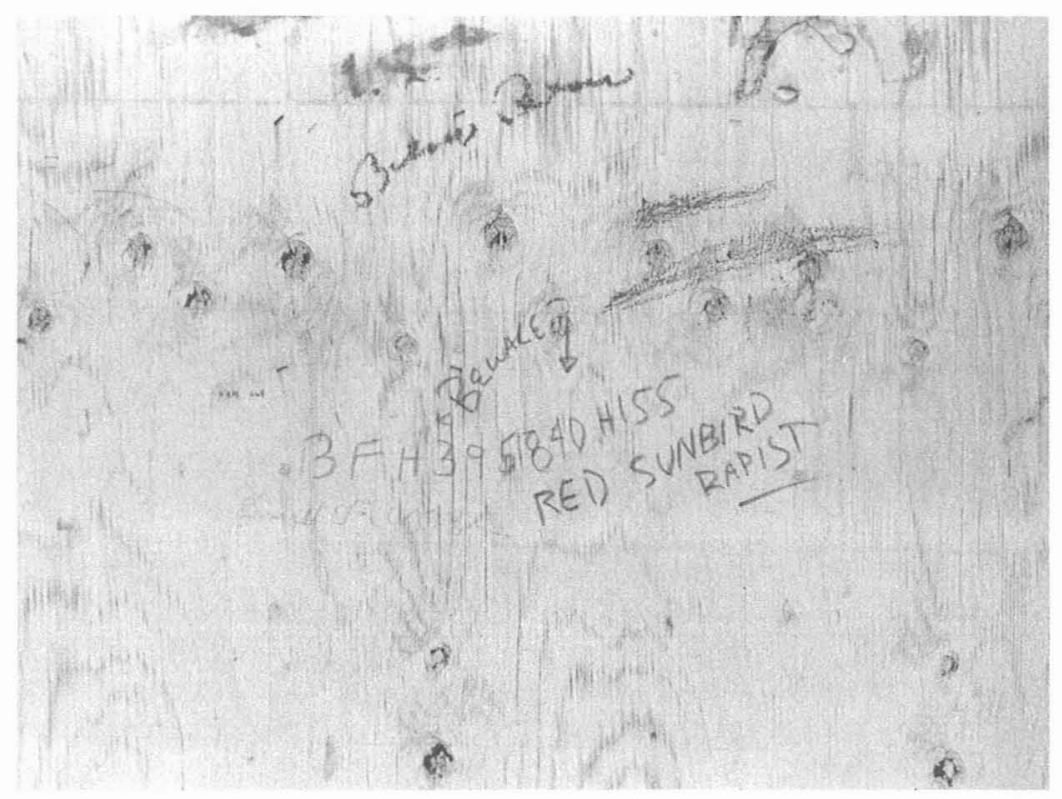

3.

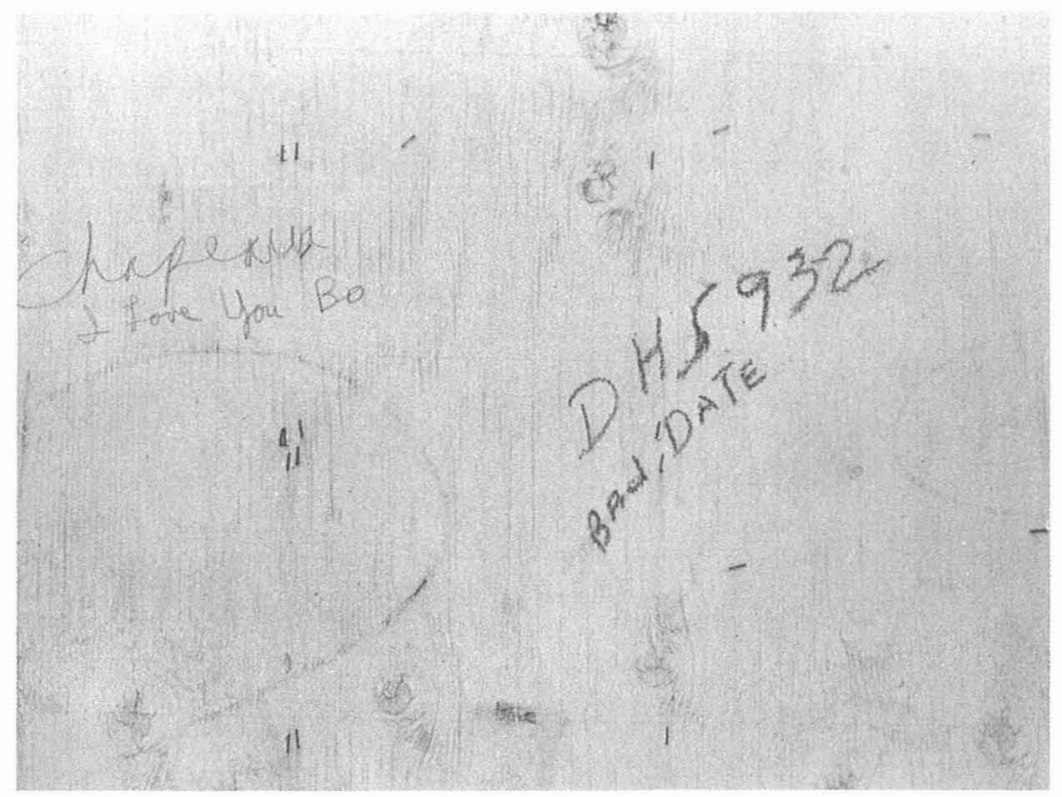

4. 
Et aurions-nous su l'interpréter sans les commentaires donnés par un informateur à la photographe de notre équipe, Josée Lambert: ces panneaux sont utilisés par les prostituées qui s'avertissent les unes les autres des dangers que des clients leur font courir dans la pratique quotidienne de leur métier. Les lire, c'est transgresser l'intimité d'un groupe particulièrement secret et Josée Lambert l'a bien senti quand. elle a tenté en vain d'aborder une fille dont elle espérait obtenir des explications supplémentaires.

Quel rôle jouent ces panneaux? En quoi les graffiti qu'ils comportent diffèrent-ils des graffiti 'classiques' (oh!)? Un seul regard sur eux suffit pour convaincre le lecteur qu'il est hors du coup, que les graffiteures l'ont exclu sans ambiguïté de la relation de communication qu'elles ont voulu établir et qu'il n'y entre que par le biais d'une sorte de voyeurisme. On ne l'insulte pas, on ne le provoque pas ainsi que le graffito se doit de le faire: on le traite comme s'iln' existait pas en lui imposant sa propre absence. De toute évidence, les graffiti qu'il décode ne lui sont pas destinés. Quant aux panneaux, ils fonctionnent tels des babillards à circuit fermé.

Voyons de plus près les photographies que Josée Lambert a réussi à prendre. Aucun excès formel: le script plus souvent qu'autrement, réalisé non pas à l'aide des moyens traditionnels - bombonne aérosol, pinceau, crayon feutre, pochoir, etc. - mais avecles instruments même (les marques?) du métier, crayon à maquillage et bâton de rouge à lèvres. L'équivalent, en somme, du message banal laissé derrière soi dans un lieu familier. Aucune tentative non plus de supplanter le discours de l'autre en le rayant ou en s'y super-posant. Aucune tentative surtout d'ouvrir la moindre polémique.

Les graffiteures se contentent d'écrire pour le bien-être (parfois la survie) des leurs consoeurs:

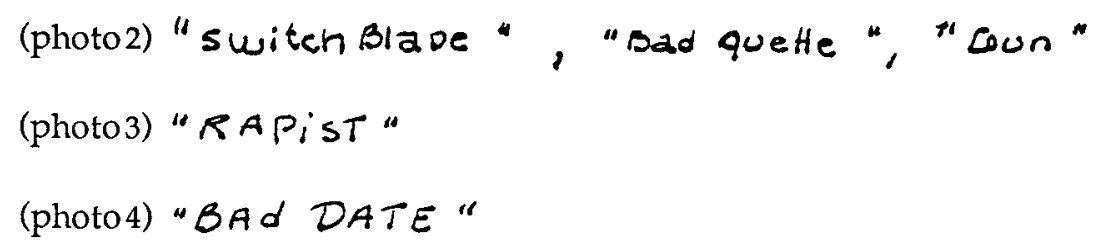




\section{Graffiti et prostitution - 111}

$\mathrm{Au}$ besoin, elles ajouteront des précisions, descriptions d'un client, marque, couleur et numéro d'immatriculation d'une voiture, numéro de téléphone même. Quand 'la réalité passe la fiction', comment le lecteur resterait-il indifférent au tragique qui affleure dans de tels graffiti? Tragique d'autant plus grand qu'il est banalisé ...

\section{Notes}

1 Ernst, L. Abel and Barbara E. Buckley, The handwriting on the Wall, Westport, Connecticut/London, England: Greenwood Press, p. 14.

2 Graffito relevé dans un stationnement montréalais, coin Bleury et SteCatherine. Cf. Montréal graffiti (Jeanne Demers, Josée Lambert, Line McMurray), Montréal: VLB éd., 1987, p. 44.

3 Montréal graffiti, pp. $71,130,18,95,93,77$.

4 Le phénomène a beaucoup été étudié. Ne citons que l'article de David Ley et Roman Cybriwsky, 'Urban Graffiti as Territorial Markers', in Annals of the Association of American Geographers, vol. 64, 1974, John Fraser éd., pp. 491-505. 